\title{
Le laser en parodontie
}

\section{RÉSUMÉ}

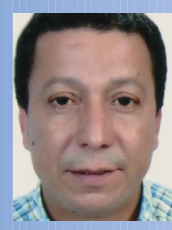

Driss BENAAZZA

Professeur agrégé en parodontologie, Faculté de médecine dentaire de Rabat.

BP 6212, Rabat Institut,

Maroc.

\section{Amine CHERKAOUI}

Professeur agrégé en parodontologie, Faculté de médecine dentaire de Rabat. Maroc.

\section{Mustapha ELMOUADDEN}

Professeur agrégé en parodontologie, Faculté de médecine dentaire de Rabat. Maroc.

\section{Bouabid ELMOHTARIM}

Professeur en prothèse adjointe,

Chef de service d'odontologie,

Hôpital militaire d'instruction Mohammed V,

Rabat, Maroc.
Le laser est actuellement très utilisé dans la recherche, l'industrie, la médecine et les technologies nouvelles. Ce développement est particulièrement marqué dans les disciplines médicales et l'odontologie n'échappe pas à ce phénomène.

L'usage chirurgical de certains lasers sur les tissus mous de la cavité buccale est connu. Cependant leur utilisation pour le traitement de la poche parodontale et pour la chirurgie de l'os alvéolaire est sujet à controverse. Après avoir rappelé les caractéristiques des différents types de lasers et leurs effets sur le tissu ciblé, nous discutons leurs applications actuelles en thérapeutique parodontale.
- laser

- effets tissulaires

- parodontie 


\section{Introduction}

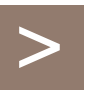

Le laser est un pur produit du vingtième siècle. C'est un faisceau lumineux au même titre que n'importe quel faisceau lumineux classique, constitué de particules de lumière appelées photons. Cependant le laser a des propriétés physiques particulières évoquées par le sigle LASER, qui signifie Light Amplification by Stimulated Emission of Radiation ou «amplification de la lumière par émission stimulée de rayonnement». Le même mot laser désigne à la fois le mécanisme et l'appareil qui fonctionne sur la base de ce mécanisme [1].

Les théories d'Einstein sur le contrôle des radiations ont été à l'origine de la technologie du laser, et son article sur l'émission stimulée de l'énergie rayonnante publié en 1917 [2] est reconnu comme la base de la notion de la lumière amplifiée.

En 1960, le premier laser a été mis au point par Maiman [3]. Un an après cet exploit, Goldman établit le premier laser médical de laboratoire. Ensuite les découvertes techniques se sont suc- cédé rapidement et, au cours des années, Goldman et d'autres chercheurs ont démontré la capacité des différents types de lasers à couper, coaguler, exciser et vaporiser les tissus biologiques [4].

Actuellement, le laser est très utilisé dans la recherche, l'industrie, la médecine et les technologies nouvelles; et s'il convient de suivre les progrès de cette technologie nouvelle, il faut cependant éviter de céder à un engouement qui n'est pas toujours justifié. Le laser n'est pas un appareil miracle pouvant apporter une solution à tout problème, mais il peut, dans certaines applications définies, être tout à fait intéressant en odontologie.

Le but de ce travail est de rappeler les caractéristiques des différents types de lasers et leurs effets sur les tissus vivants, puis de discuter leurs applications cliniques actuelles en parodontie. Cette connaissance est indispensable à un acte thérapeutique de qualité réalisé dans des conditions de sécurité parfaite.

\section{Classification des lasers}

Les différents types de lasers peuvent être classés selon plusieurs paramètres:

- la longueur d'onde, qui est généralement indiquée en nanomètres $(\mathrm{nm})$ ou en micromètres $(\mu \mathrm{m})$ pour les lasers médicaux ou dentaires (tableau I) $[5,6]$;

- le mode d'émission, qui peut être [6] :

- continu : dont on peut faire varier la puissance et la fréquence d'émission,

- pulsé normal : très courtes impulsions (pulses), entrecoupées de périodes de repos,
- pulsé déclenché : impulsions brèves et très intenses. La puissance de crête (énergie par durée moyenne d'impulsion) est supérieure à celle obtenue en mode pulsé normal ;

- le matériau actif qui génère le rayonnement : il peut être un solide, un liquide, un gaz ou un mélange gazeux, ou des semiconducteurs [6] :

- la puissance exprimée en watts, on distingue alors [6] :

- les lasers de forte puissance caractérisés par leur effet thermique, utilisés en chirurgie, 
tels que le laser Nd-YAG et le laser à gaz carbonique $\left(\mathrm{CO}_{2}\right)$,

- les lasers de moyenne puissance à effet photochimique et thermique, tel que le laser diode,
- les lasers de faible puissance ou lasers athermiques dits «soft lasers», tel que le laser hélium néon ( $\mathrm{He} \mathrm{Ne}$ ).

Tableau I

Classification des lasers selon la longueur d'onde [5, 7].

\begin{tabular}{lcc}
\hline \multicolumn{1}{c}{ Type de laser } & Couleur & $\begin{array}{c}\text { Longueur d'onde } \\
(\mathbf{n m})\end{array}$ \\
\hline $\begin{array}{l}\text { Excimer : fluorure d'argon } \\
\text { ou chlorure de xénon }\end{array}$ & Ultra-violet & 193 \\
\hline Argon & Ultra-violet & 348 \\
\hline Dioxyde de carbone $\left(\mathrm{CO}_{2}\right)$ & Bleu & 488 \\
\hline Hélium-néon (HeNe) & Ileu/vert & 515 \\
\hline Rubis (cristal d'alumine) & Rouge & 10600 \\
\hline $\begin{array}{l}\text { Nd-Yag (grenat d'yttrium-aluminium } \\
\text { dopé au néodyme) }\end{array}$ & Rouge intense & 630 \\
\hline $\begin{array}{l}\text { Er-Yag (grenat d'yttrium-aluminium } \\
\text { dopé à l'erbium) }\end{array}$ & Infrarouge & 1064 \\
\hline $\begin{array}{l}\text { Lasers diodes (semi-conducteurs) : tel que } \\
\text { l'arséniure de gallium }\end{array}$ & Infrarouge & 2940 \\
\hline
\end{tabular}

\section{Effets de l'énergie laser sur les tissus}

Les phénomènes rencontrés lors de l'action du laser sur la matière vivante sont dominés par l'effet thermique dont la coagulation et la volatilisation (effet de coupe) constituent les principales particularités de la chirurgie laser.

L'effet thermique se définit comme l'absorption énergique globale du tissu irradié et sa dégradation locale en chaleur, il correspond successivement aux étapes de température suivantes : échauffement $\left(37^{\circ}-60^{\circ} \mathrm{C}\right)$, soudure $(60$ $\left.65^{\circ} \mathrm{C}\right)$, coagulation $\left(65-90^{\circ} \mathrm{C}\right)$, dénaturation de protéines $\left(90-100^{\circ} \mathrm{C}\right)$, volatilisation ou vaporisation (à $100^{\circ} \mathrm{C}$ ), au-delà de $100^{\circ} \mathrm{C}$, c'est la carbonisation [7].
Chaque tissu présente des propriétés thermiques particulières, l'énergie absorbée est également fonction de la longueur d'onde de chaque type de laser. Ces paramètres jouent un rôle important que le clinicien doit parfaitement comprendre et pouvoir maîtriser. Quand le rayon laser atteint un tissu, il peut être réfléchi, dispersé, absorbé ou transmis vers les tissus environnants [8].

Une meilleure connaissance des interactions laser-tissu en milieu buccal permet de sélectionner les effets recherchés en minimisant les effets secondaires sur les tissus cibles et les structures adjacentes. 


\section{Application des lasers en parodontie}

Les premières utilisations du laser en odontologie avaient pour but de remplacer le fraisage mécanique pour l'élimination des caries et la préparation des cavités. C'est ainsi qu'après la découverte du laser à rubis en 1960, Goldman et al. [4] en 1964 I'ont essayé in vitro, pour l'élimination des caries. Depuis, plusieurs recherches ont été menées sur les effets des différents lasers comme l'argon, le $\mathrm{CO}_{2}$ et le $\mathrm{Nd}-\mathrm{YAG}$, sur les tissus dentaires durs et les caries [9]. En parodontie, la première étape thérapeutique importante est constituée par le débridement mécanique non chirurgical des surfaces radiculaires, et déjà en 1965 Kinersly et al. rapportaient la possibilité d'éliminer le tartre dentaire par le laser à rubis, lors de cette étape ; mais le problème était de limiter sélectivement la vaporisation du tartre sans endommager la dent sousjacente [10]. Pour les tissus parodontaux mous, certains lasers utilisés à haute puissance, comme le $\mathrm{CO}_{2}$ et le $\mathrm{Nd}-\mathrm{YAG}$, ont une excellente capacité de leur ablation, et ils étaient approuvés depuis quelques années pour la gestion de ces tissus ainsi que pour la chirurgie orale[7, 11, 12]. Jusqu'au début des années 1990, I'utilisation des lasers en parodontie était limitée aux tissus mous comme la gingivectomie et la frénectomie [13], et leurs utilisations sur les surfaces radiculaires et sur l'os alvéolaire n'étaient pas utiles à cause de leurs effets thermiques secondaires sur les tissus ciblés et de voisinage.

Quelque temps après, des recherches scientifiques ont commencé sur l'usage du laser $\mathrm{Nd}$ YAG pour le débridement des surfaces radiculaires et le curetage des poches parodontales ; en effet, la souplesse et la maniabilité de sa fibre optique le rendent très approprié à cet usage.

\section{Application sur les tissus durs}

Plusieurs études ont montré l'effet négatif du laser $\mathrm{CO}_{2}$ tel que la carbonisation, quand il est appliqué directement sur les tissus durs; son usage était limité aux procédures des tissus mous [14]. Cependant la nouvelle fibre flexible délivrée avec ce laser peut rendre possible son usage dans les poches parodontales dans un futur proche. En effet, certaines études indiquent que I'usage de ce laser avec une puissance élevée et en mode continu n'est pas approprié pour le détartrage et le débridement des surfaces radiculaires à cause des effets thermiques secondaires; mais, à une puissance relativement basse et en mode pulsé et/ou défocalisé, il peut être utilisé sur la sur- face radiculaire contaminée avec des effets bactéricides et de détoxication, sans endommager la surface radiculaire [15]. Pour Crespi et al. [16], le laser $\mathrm{CO}_{2}$ en mode pulsé défocalisé est un moyen utile pour le conditionnement de la surface radiculaire quand il est combiné à l'instrumentation mécanique. Cependant, jusqu'à présent, aucune étude clinique ne rapporte l'application du laser $\mathrm{CO}_{2}$ dans la poche parodontale [5].

Pour le laser Nd-YAG, les opinions diffèrent quant à son utilisation pour le traitement des poches parodontales. En 1997, I'US Food and Drug Administration (FDA) a approuvé son application en mode pulsé pour le débride- 
ment sulculaire ou le curetage des tissus mous [17]. C'était la première approbation par la FDA du laser pour le traitement de la poche parodontale. Le laser Nd-YAG a également reçu l'agrément de I'Académie of Laser Dentistry (ALD), comme adjuvant du débridement mécanique des surfaces radiculaires [18]. D'autres études antérieures, réalisées in vitro et in vivo ont montré l'efficacité de ce laser pour l'élimination complète du tartre sans dommages thermiques [19], et la réduction ou l'inhibition de la recolonisation bactérienne. La couleur foncée du tartre sous-gingival constitue un avantage pour le laser Nd-YAG, vu sa grande absorption par des zones plus sombres [20]. Cependant d'autres études rapportent que l'élimination des dépôts calcifiés n'est que partielle, avec des dommages thermiques sur la surface radiculaire [21].

En fait, s'il est utilisé, le laser Nd-YAG devrait l'être avec une puissance d'énergie relativement basse et comme adjuvant du traitement mécanique conventionnel, plutôt que comme un traitement primaire de la poche parodontale. Il permettra aussi bien le curetage des tissus de granulation infectés que la désinfection et la détoxication des poches parodontales, plutôt qu'un débridement des dépôts calcifiés et du cément contaminé par une énergie élevée avec risque de produire des dommages thermiques [5].

Le laser Er-YAG a été introduit en 1974 par Zharikov et al. [22], il possède un coefficient d'absorption pour l'eau très supérieur à celui du $\mathrm{CO}_{2}$ et du Nd-YAG. Il est ainsi bien absorbé par tous les tissus biologiques dont les molécules contiennent de l'eau. La capacité d'ablation des tissus dentaires durs par le laser ErYAG, sans effets thermiques secondaires, est décrite par certaines études [23].

L'utilisation du laser Er-YAG en mode pulsé a reçu l'agrément de la FDA pour la chirurgie des tissus mous et le débridement sulculaire en 1999, et pour la chirurgie osseuse en 2004 [24]. D'autres études menées jusqu'à présent indiquent la sécurité et l'efficacité de l'application clinique du laser Er-YAG pour le traitement des poches parodontales, y compris le débridement des surfaces radiculaires, avec des effets bactéricides, ainsi que la dégradation et l'élimination des endotoxines bactériennes [25]. Ce laser possède également la capacité d'ablation osseuse suivie d'une bonne cicatrisation, il pourrait être cliniquement appliqué pour la chirurgie osseuse en parodontie [12].

\section{Application sur les tissus mous}

L'utilisation des lasers $\mathrm{CO}_{2}$ et $\mathrm{Nd}$-YAG, pour la chirurgie buccale en général et les tissus parodontaux mous en particulier, a été largement approuvée [7], grâce à leur excellente capacité d'ablation des tissus mous accompagnée de grands effets hémostatiques et bactéricides [13, 26]. Ils étaient les premiers lasers à avoir des pièces à main adaptées à l'usage intra-oral.
Le laser $\mathrm{CO}_{2}$, utilisé en mode pulsé ou continu, est très fortement absorbé par l'eau. Et comme les tissus mous sont composés de 75 à $90 \%$ d'eau, environ $98 \%$ de l'énergie laser est convertie en chaleur et absorbée en surface de ces tissus, avec très peu de pénétration ou de dispersion [9]. Une très petite contraction de la plaie et une cicatrice minime sont d'autres avantages de la chirurgie laser, et en particu- 
lier le $\mathrm{CO}_{2}$. Le laser $\mathrm{CO}_{2}$ a été utilisé pour la chirurgie des tissus mous depuis 1970 [27]. En 1976, il a reçu l'agrément de la FDA pour la chirurgie des tissus mous, y compris la chirurgie des tissus oraux.

L'usage chirurgical du laser Nd-YAG en mode pulsé a été approuvé en 1990 par la FDA [24]. Il est très peu absorbé par l'eau, son énergie se disperse ou pénètre dans les tissus biologiques, avec une profondeur estimée à $2+$ ou $-1 \mathrm{~mm}$ (pour les tissus mous) [9]. De par sa grande absorption par les zones plus sombres, l'encre indien et d'autres substances à pigment noir sont souvent appliquées sur le tissu cible pour augmenter I'efficacité de I'ablation [20]. Le laser Nd-YAG est de ce fait idéal pour ablation des lésions tissulaires hémorragiques et pour I'hémostase des petits capillaires et des veinules, il peut coaguler des vaisseaux sanguins jusqu'à $1 \mathrm{~mm}$ de diamètre au lieu de 0,5 mm pour le laser $\mathrm{CO}_{2}$. Cependant l'effet de dispersion diminue la pénétration du rayonnement, notamment dans les tissus de couleur pâle [7]. Les lasers diodes sont des lasers semi-conducteurs, émis en mode continu ou pulsé, dotés d'une fibre optique flexible. Leur absorption par l'eau est faible, alors qu'ils sont très absorbés par l'hémoglobine et d'autres pigments [15]. Le laser diode n'interagit pas avec les tissus dentaires durs, mais il est excellent pour la chirurgie des tissus mous, indiqué pour couper, coaguler la gencive et la muqueuse orale, et pour le curetage des tissus mous ou le débridement [28]. Son usage pour tissus mous a été approuvé par la FDA en 1995, et en 1998 pour le débridement sulculaire. La pénétration tissulaire du laser diode est inférieure à celle du Nd-YAG, alors que la vitesse d'échauffement est supérieure [29], entraînant une profonde coagulation et plus de calcination en surface si on le compare au laser Nd-YAG. La dimension réduite et le coût moins élevé sont d'autres avantages de ce laser.

Le laser argon, émis en modes continu ou pulsé, est très peu absorbé par l'eau et par conséquent il n'interagit pas avec les tissus durs. Cependant il est bien absorbé par les tissus pigmentés, y compris l'hémoglobine, la mélanine et les bactéries pigmentées. Bien qu'il ne soit pas largement utilisé en thérapeutique parodontale, il est communément utilisé pour polymérisation des composites et blanchiment au cabinet dentaire. Ce laser a reçu l'agrément de la FDA pour la chirurgie des tissus mous et la polymérisation des composites en 1991, et pour le blanchiment des dents en 1995 [17].

Plusieurs concepteurs de lasers recommandent et soulignent leurs «avantages» par rapport aux thérapeutiques classiques dans les indications suivantes :

- décontamination des poches parodontales ;

- traitements chirurgicaux tels que : curetage gingival, gingivectomie-gingivoplastie, frénectomie [30].

Ainsi, en plus des avantages hémostatique et bactéricide, l'exploitation du laser permet un champ opératoire toujours dégagé et une visibilité meilleure avec un temps d'intervention réduit. Les suites opératoires sont généralement faibles et la douleur est atténuée grâce à la technique qui est moins traumatisante car le laser volatilise les tissus au lieu de les dilacérer. Par ailleurs, la cicatrisation après traitement au laser est plus longue qu'après une chirurgie classique [13]. George Krygier [31] pour sa part souligne les résultats très encourageants obtenus avec le laser Nd-YAG et insiste sur la maniabilité des systèmes commercialisés à I'heure actuelle dont la compacité et la souplesse de la fibre optique en font des appareils d'utilisation pratique au cabinet dentaire. II 
considère que les indications du laser sont extrêmement larges et vont de la décontamination des poches au geste chirurgical, en passant par le traitement des aphtes.

Dans son rapport de 2002 [7], I'Académie américaine de parodontologie (AAP) recommande les lasers $\mathrm{CO}_{2}$ et $\mathrm{Nd}-\mathrm{YAG}$, ainsi que le laser diode [32] pour certaines indications.

Le laser $\mathrm{CO}_{2}$ est recommandé pour : la gingivectomie, la frénectomie et l'excision des tissus pathologiques mous (bénins ou malins), ainsi que pour la désépithélialisation des lambeaux muco-périostés au cours de la chirurgie et après chirurgie [33].

Le laser Nd-YAG est recommandé pour : la gingivectomie, la frénectomie, l'excision des tissus pathologiques mous, particulièrement les lésions hémorragiques, il est également utilisé pour le curetage sous-gingival des poches parodontales. Les applications cliniques du laser diode sont similaires à celles du Nd-YAG [34].

Nous décrivons ci-après les techniques opératoires de quelques indications thérapeutiques du laser, telles que le traitement des poches parodontales, la frénectomie, et l'ablation des épulis ou fibromes, pour lesquelles nous avons utilisé le laser Nd-YAG.

\section{Traitement}

des poches parodontales (fréquence $(\mathrm{f})=10$ à $20 \mathrm{~Hz}$, puissance $(\mathrm{P})=1,5$ à $2,25 \mathrm{~W})$

On réalise une détoxication associée à une désinfection : la fibre du laser Nd-YAG est insérée jusqu'au fond de la poche parallèlement à la surface de la racine dentaire et reste au contact permanent avec les tissus mous et les tissus durs (cément) éliminant les bactéries sans altérer le cément [1] (fig. 1). Le pouvoir de stérilisation du rayon laser permet dans un même temps l'élimination des endotoxines et la destruction des germes présents au niveau des porosités cémentaires. Le temps opératoire est court (une minute par dent), au rythme de deux applications par séance, quatre applications au total suffisent pour obtenir un résultat spectaculaire[1].

\section{Frénectomie $(\mathrm{f}=20$ à $30 \mathrm{~Hz}$, $\mathrm{P}=1,75$ à $3 \mathrm{~W}$ )}

C'est peut-être l'une des plus spectaculaires applications du laser en parodontie, et l'acte le plus facile. Dans les méthodes classiques, on a

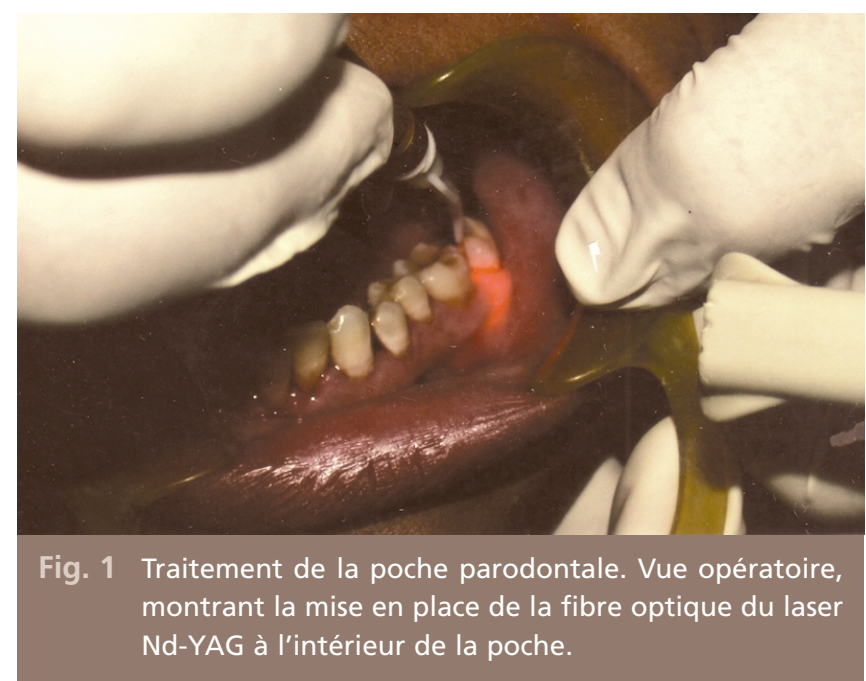



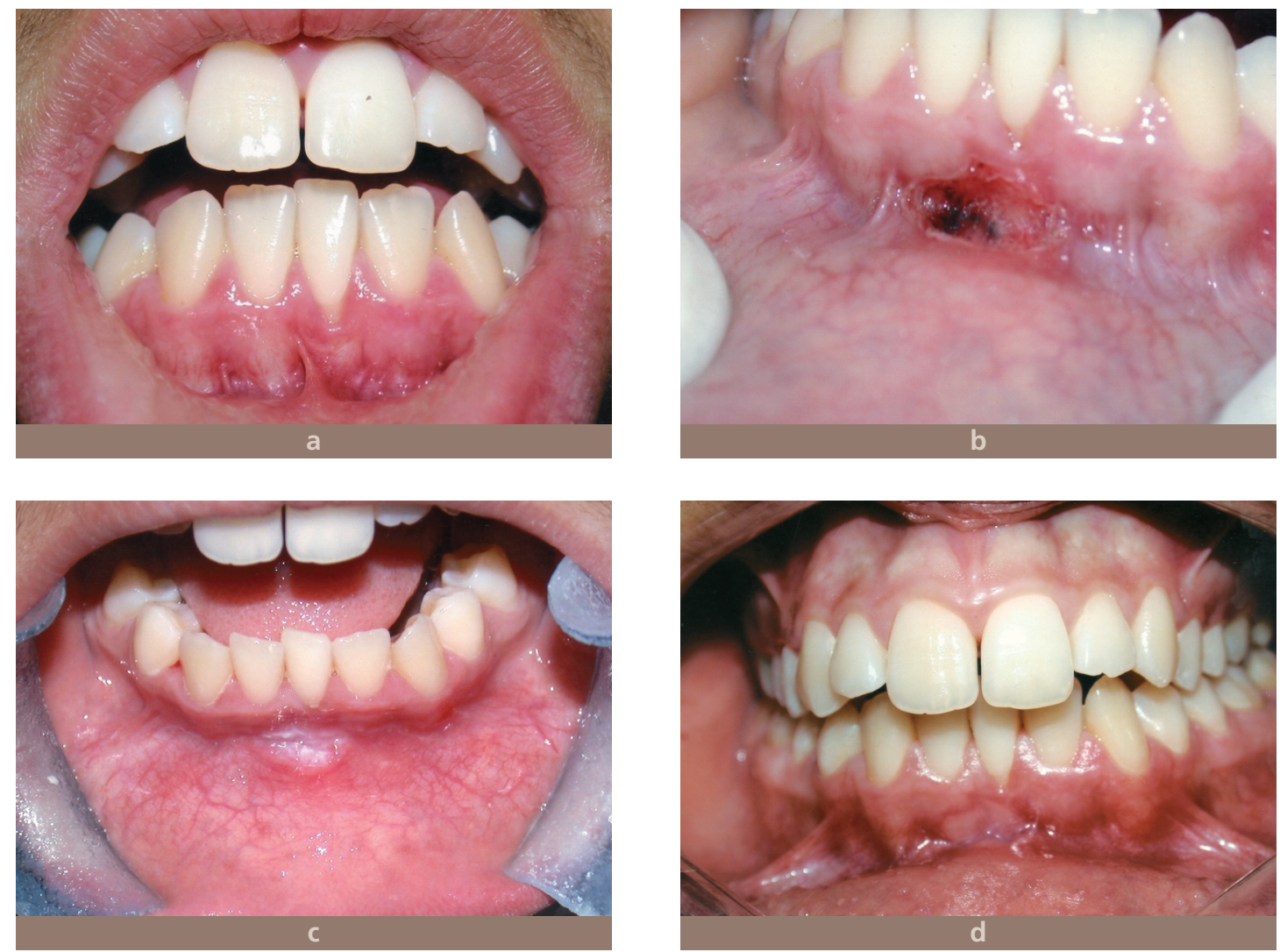

Fig. 2 a à d Frénectomie labiale, au laser Nd-YAG.

a : Frein pathologique, avec récession gingivale au niveau de 31.

b : Vue postopératoire immédiate.

c: Cicatrisation après une semaine (plaie d'aspect blanchâtre caractéristique).

$d$ : Bonne cicatrisation après un mois, avec persistance de légères tâches blanches.

toujours besoin d'une anesthésie profonde, d'une lame, et de sutures, I'hémorragie est considérable au cours de l'intervention et les suites opératoires sont complètement inconfortables. Alors que la frénectomie au laser est exsangue, avec le minimum d'anesthésie, sans sutures et indolore et des suites opératoires très simples [35]. La section de freins labiaux supérieurs et inférieurs, ainsi que du frein lingual, peut être facilement réalisée, même sur des enfants de moins de 6 ans. Le frein mis en tension est sectionné par le laser de façon tangentielle [1, 35] (fig. 2 a à d).
Ablation de tissus

pathologiques mous

(tels que les épulis)

(f $=20$ à $30 \mathrm{~Hz}$,

$\mathrm{P}=1,75$ à $3 \mathrm{~W}$

(maximum $5 \mathrm{~W}$ )

Ils sont facilement excisés à l'aide du laser qui permet une ablation tissulaire totalement exsangue. À une puissance de 1 watt, le praticien vaporise les tissus visés, tenus en traction et contre-traction, tout en évitant la carboni- 

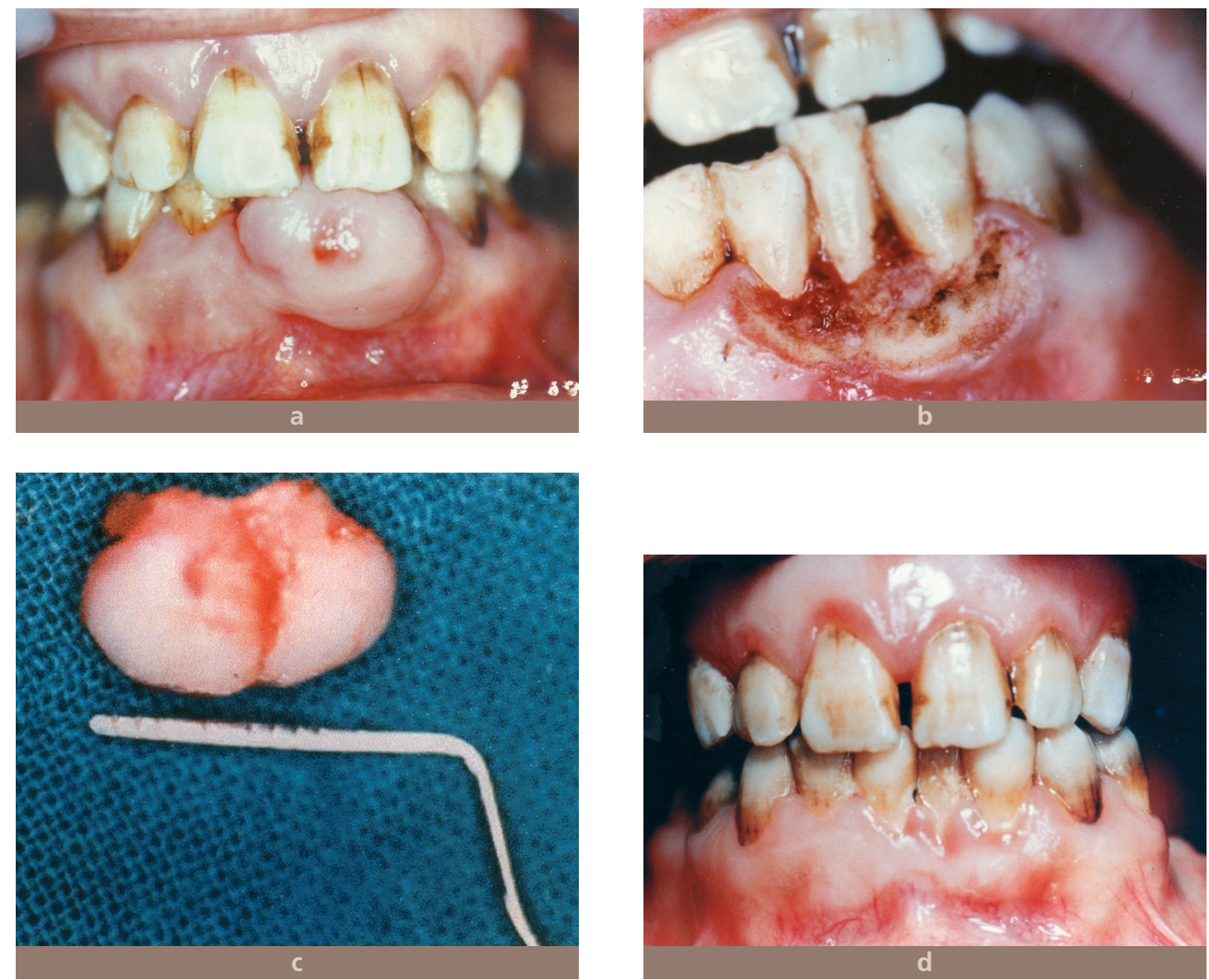

Fig. 3 a à d Excision d'épulis au laser Nd-YAG.

a : Vue préopératoire d'une épulis s'étendant de 32 à 41 . Noter la fluorose très prononcée chez cette patiente.

b : Vue postopératoire immédiate (noter l'excision totale de la tumeur, avec bonne hémostase), le site est couvert par un pansement chirurgical pendant une semaine.

c : Pièce opératoire (environ 14 mm sur 8).

$\mathrm{d}$ : Cicatrisation après un mois.

sation du site par nettoyage avec du sérum physiologique après chaque passage du laser. La reépithélisation débutera dès le $14^{\mathrm{e}}$ jour et sera complète au bout de 4 semaines [1]

(fig. 3 a à d). 


\section{Inconvénients des lasers et précaution à prendre en utilisation clinique}

Le laser est peut-être un moyen nouveau et efficace en thérapeutique parodontale. Cependant il a aussi bien des avantages que des inconvénients et certaines précautions doivent être prises lors de son utilisation [7] (tableau II).

\section{Tableau II}

Inconvénients des lasers et précautions à prendre en utilisations cliniques.

Précautions avant et pendant l'irradiation

Port de lunettes de protection (patient, opérateur et assistants).

Port de gants et masques (opérateur et assistants) (fig. 4).

Aspiration chirurgicale continue, pour réduire l'odeur de brûlure, ainsi que les fumées et débris issus des lésions traitées.

Risque de destruction tissulaire excessive par ablation directe et effets thermiques secondaires

Destruction de l'appareil d'attache au fond de la poche.

Ablation excessive des surfaces radiculaires et des tissus gingivaux à l'intérieur de la poche.

Lésions thermiques au niveau de la surface radiculaire, des tissus gingivaux, de la pulpe, et du tissu osseux.

Inconvénient lié aux systèmes lasers

Prix élevés des appareils.

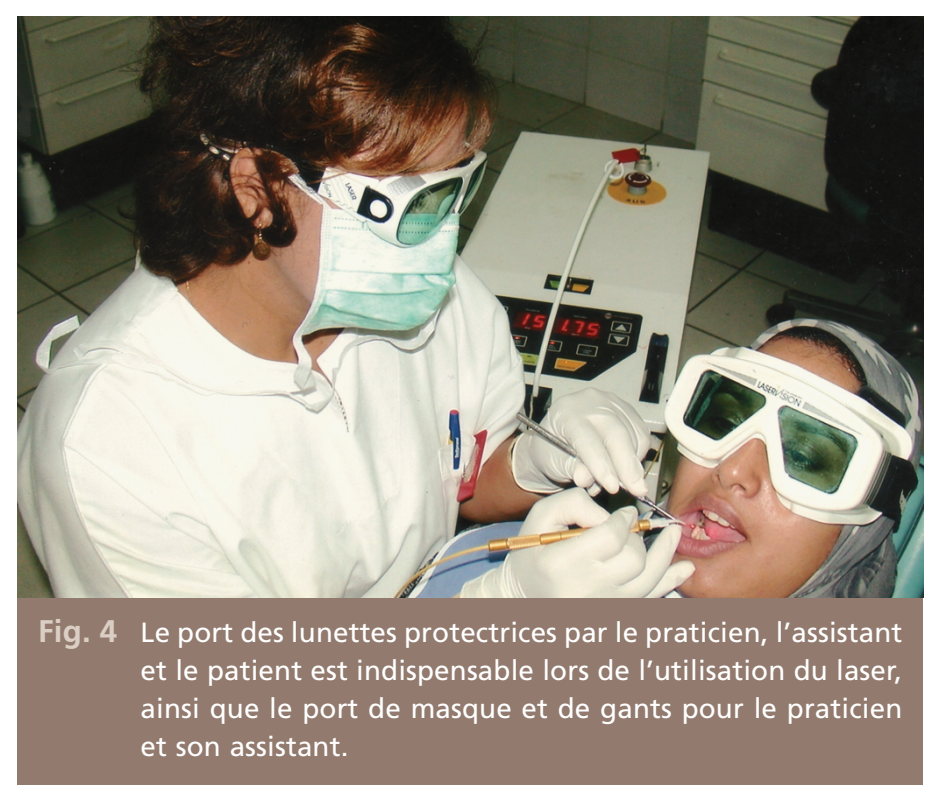




\section{Conclusion}

À cause de certaines limites des thérapeutiques parodontales conventionnelles, I'utilisation des lasers comme moyen adjuvant ou alternatif de celles-ci ouvre de nouveaux horizons en pratique parodontale. Leur utilisation doit être bien définie et les indications de chacun d'eux sont spécifiques. Elles diffèrent selon la nature du tissu et la densité d'énergie trans- mise par unité de surface. De bons résultats ne peuvent être obtenus que si le rayonnement laser est utilisé à bon escient, avec les connaissances requises.

La technique laser prend peu à peu le pas sur les systèmes mécaniques, son avenir peut s'avérer immense.

\section{Bibliographie}

1. Thiria $C$.

Le choix laser.

Inf Dent 1997:42:3227-3232.

2. Einstein $\mathrm{A}$.

Zur quantum theorie

der strhlung.

Physikalische zeitschift 1917;18:121-128.

3. Maiman $\mathrm{TH}$. Stimulated optical radiation in ruby lasers. Nature 1960;187:493-494.

4. Goldman L, Hornby $P$, Myer R, Goldman B. Impact of the laser on dental caries. Nature 1964;25:417.

5. Aoki A, Miyuki SK, Watanabe $\mathrm{H}$, Ishikawa I. Lasers in nonsurgical periodontal therapy. Periodontol 2000 2004;36:59-97.

6. Franquin JC, Coche $P$, Salomon JP. Principes d'utilisation clinique du laser hélium-néon en odontologie. L.Q.O.S. 1986;11:217-231.

7. AAP (The American Academy of Periodontology).
The research Science and therapy committee of the American Academy of Periodontology, Cohen RE, Ammons WF. Revised by Rossman JA. Lasers in Periodontics (Academy report). J Periodontol 2002;73:1231-1239.

8. Niemz $\mathrm{MH}$.

Laser tissue interaction.

Fundamentals and applications. Berlin: Springer-Verlag 1996:64-65.

9. Haldorsson T, Langerholk J. Thermodynamic analysis of laser irradiation of biological tissue. Appl Opt 1978;17:3948.

10. Kinersly, Jarabak JP, Phatak NM, DeMent J. Laser effects on tissue and materials related to dentistry.

J Am Dent Assoc 1965;70:593-600.

11. ALD (The Academy of Laser Dentistry). Featured wavelength: diode - the diode laser in dentistry

(Academy report).

Wavelengths 2000;8:13.

12. Aoki A, et al.

Comparative study of Er-YAG laser and rotating bur for bone ablation. In: Ishikawa I, Frame JW, Aoki A, editors. Lasers in Dentistry - Revolution of Dental Treatment in the new millennium. Amsterdam: Elsevier Science 2003:389-391.

13. Pick RM, Colvard MD. Current status of lasers in soft tissue dental surgery. J Periodontol 1993;64:589-602.

14. Lobene RR, Bhussry BR, Fine $\mathrm{S}$.

Interaction

of carbon dioxide laser radiation with enamel and dentin.

J Dent Res 1968;47:311-317.

15. Barone A, Covani U, Crespi R, Romanos GE.

Root surface morphological changes after focused 
versus defocused $\mathrm{CO}_{2}$ laser irradiation: a scanning electron microscopy analysis.

J Periodontol 2002;73:370-373.

16. Crespi R, Barone A, Covani U, Ciaglia RN, Romanos GE. Effects of $\mathrm{CO}_{2}$ laser treatment on fibroblast attachment to root surfaces. A scanning electron microscopy analysis. J Periodontol 2000;73:1308-1312.

17. Sulewski JG. Historial survey of laser dentistry. Dent Clin North Am 2000;44:717-752.

18. Coluzzi DJ. Laser assisted sulcular debridement.

Featured wavelength: Nd-YAG.

Wavelengths 2001;9:19.

19. Arcoria CJ, Vitasek-Arcoria BA. The effects of low level energy density Nd-YAG irradiation on calculus removal.

J Clin Laser Med Surg 1992;10:343-347.

20. Jennet E, Motamedi M, Rastegar S, Frederickson C, Arcoria C, Powers JM. Dye-enhanced ablation of enamel by pulsed lasers. J Dent Res 1994;73:1841-1847.

21. Tseng $P$, Liew $V$.

The use of a Nd-YAG dental laser in periodontal therapy. Aust Dent Assoc News Bull 1991;Nov: 3-6.

22. Zharikov EV, Zhekov VI, Kulevskii LA, et al. Stimulated emission from $\mathrm{Er} 3+$ ions in yttrium aluminum garnet crystals at $?=2.94 \mu$.
Sov J Quantum Electron 1975;4:1039-1040.

23. Hibst R, Keller U. Experimental studies of the application of the R-YAG laser on dental hard substances.

I. Measurement of the ablation rate.

Lasers Surg Med 1989;9:338-344.

24. Keller $U$, Maier A, Paulus $R$, Hibst R.

Fluoreszenspectroscopisch Kontrolle von hurzeloberflächen nach Reinigung mit dem Er-YAG laser

Dtsch Zahnarztl Z 2001;56:481-484.

25. Folwaczny $M$, Aggstaller $H$, Hickel R.

Anti microbial effects of $\mathbf{2 . 9 4}$ micron Er-YAG laser radiation on root surfaces: an in vitro study.

$\mathrm{J}$ Clin Periodontol 2002:29:73-78.

26. Shultz RJ, Harvey GP, Fernandez-Beros $\mathrm{ME}_{\mathrm{a}}$ Krishnamurty $S$, et al. Bactericidal effects of the neodymium-YAG laser: in vitro study.

Lasers Surg Med 1986;6:445-448.

27. Pacaro BC, Garehim WJ. The $\mathrm{CO}_{2}$ laser in oral and maxillofacial surgery. J Oral Maxillofac Surg 1983;41:725-728.

28. Romanos G, Nentwig GH. Diode laser $(980 \mathrm{~nm})$ in oral and maxillofacial surgical procedures: clinical observations based on clinical applications. J Clin Laser Med Surg 1999;17:193-197.

29. Restegar $S$, Jacques $S L$, Motamedi M, Kim BM. Theoretical analysis of equivalency

of high-power diode laser and Nd-YAG laser

for coagulation.

Poc SPIE 1992;1646:150-160.

30. Epstein SR.

The frenectomy:

a comparison of classic versus laser technique. Pratical.

Periodont. Aesthetics 1991;3:27-28.

31. Conférence de la société Parisienne de Parodontologie du 17 juin avec les Professeurs Abadie MJM et Krygier G. Inf. Dent 1993;31:2280-2281.

32. Wyman A, Duffy $S$, Sweetland HM, Sharp F Rogers K.

Preliminary evaluation of a new high power diode laser.

Lasers Surg Med 1992;12:506-509.

33. Centty IG, Blank LW, Levy BA, et al.

Carbon dioxide laser for the de-epithelialization of periodontal flaps. J Periodontol 1997;68:763-769.

34. Rastegar $S$, Jacques $S L$, Motamedi M, Kim BM. Theoretical analysis of equivalency of high power diode laser and ND-YAG laser for coagulation of tissue: predictions for prostate coagulation. SPIE 1992;1646:150-160.

35. Ferrari J, Zeboulon S. Traitement thermique et chirurgie muco-gingivale : la frénectomie par micro plasma system. Inf Dent 1991;33:2821-2824. 


\section{SUMMARY}

\section{Laser in periodontics}

Driss BENAAZZA,

Amine CHERKAOUI,

Mustapha ELMOUADDEN,

Bouabid ELMOHTARIM

Keywords

- laser

- effects on tissue

- periodontics
The laser is currently very used in research, industry, medicine and the new technologies. This development is especially marked in the medical disciplines, and dentistry does not escape to this phenomenon. The surgical use of some lasers on the soft tissue of buccal cavity is known. However their use for the treatment of periodontal pocket and for the surgery of the alveolar bone is subject to controversy. After having recalled features of the different types of lasers, and their effects on the targeted tissue, we discuss their present applications in therapeutic periodontal therapy.

\section{Questions - Réponses}

1/ Réponse par vrai ou faux

a - L'utilisation du laser exige certaines précautions à prendre par le praticien et le patient

Vrai $\square \quad$ Faux

b - Parmi les inconvénients des lasers utilisés en chirurgie buccale, il y a l'hémorragie et les douleurs postopératoires

Vrai $\square \quad$ Faux

2/ QCM à une seule réponse

A - Les lasers sont classés selon :

a - l'effet bactéricide

b - la longueur d'onde

c - l'effet tissulaire

B - En clinique buccale, le laser est très utilisé :

a - pour le traitement de la carie dentaire

b - dans la chirurgie osseuse

c - comme traitement prophylactique

d - dans la chirurgie des tissus mous 


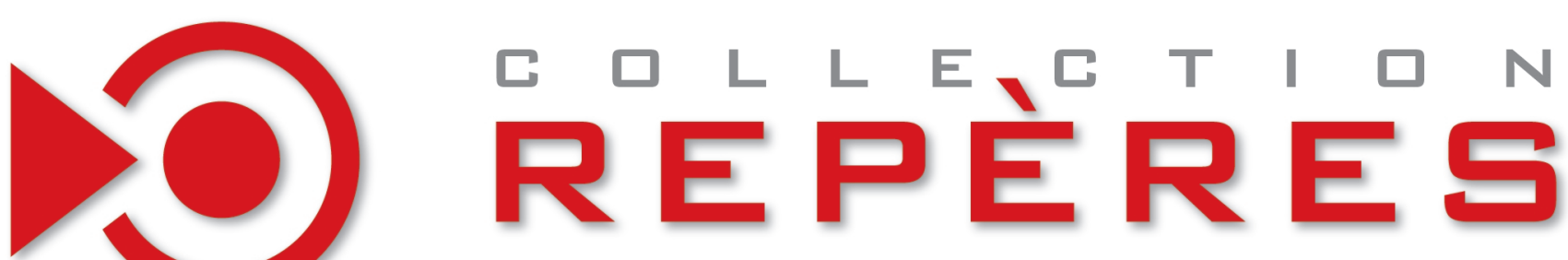

\section{Diagnostic radiologique dentaire et facial Exercices}

\section{G. Pasquet, R. Cavézian, G. Bel, G. Baller, F. Pujol, J. Batard, O. Casassus-Builhe, D. Potevin}

Ce livre propose une aide méthodologique pour l'analyse des radiographies panoramiques. Ce manuel est destiné en priorité aux étudiants de troisième cycle en orthodontie pour lesquels une connaissance approfondie de l'anatomie et de la sémiologie radiologiques craniofaciales et dentaires est un pré-requis indispensable.

200 pages - Format $24 \times 17$

Prix : $70 €$

ISBN 978-2-905302-47-X

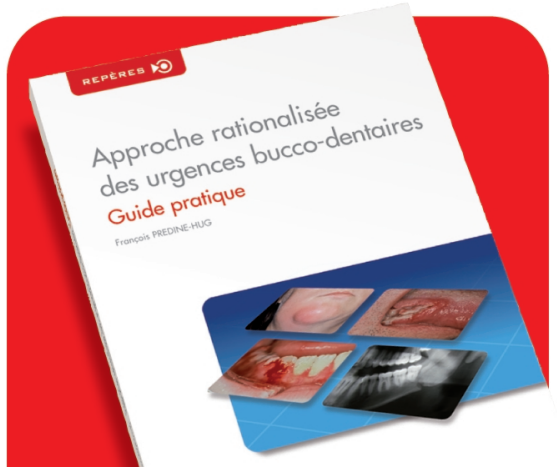

\section{Approche rationalisée} des urgences bucco-dentaires Guide pratique

\section{F. Prédine-Hug}

L'urgence peut prendre plusieurs aspects : lorsque les fonctions vitales sont compromises, la notion de temps est primordiale, mais elle peut aussi correspondre à la première décision face à une situation pathologique plus ou moins grave. De cette première décision découle une série d'actions en cascade. Une mauvaise orientation initiale ne peut qu'avoir des conséquences néfastes qu'il est très difficile de corriger par la suite. Cet ouvrage, pragmatique, de consultation simple et rapide a pour but d'aider à cette première décision.

136 pages - Format $21 \times 29,7$.

Prix : $90 €$

ISBN 978-2-7598-0449-8

Bon de commande à renvoyer aux Éditions SID, 9 rue Christine, 75006 Paris - France

Editions 50

Groupe EDP Sciences

$\nabla$ Tél : 0143293101 - Fax : 0143293262 - http://www.editionsid.com

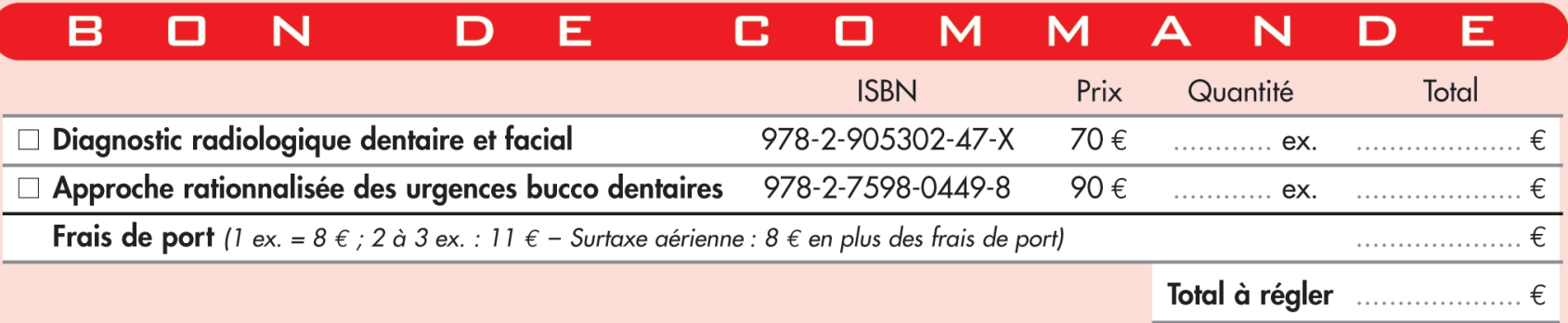

CI-JOINT, VOTRE RÈGLEMENT :

Expire :

Noter ici les trois derniers chiffres situés au dos de la carte : 1 L _ | | Signature obligatoire :

$\square$ Par chèque (à l'ordre de Éditions S.I.D.)

VOTRE ADRESSE :

Nom :

Prénom

Adresse :

Code postal : Ville : Pays : 